\title{
Trifluridine/tipiracil: an emerging strategy for the management of gastrointestinal cancers
}

\author{
Marc Peeters*,1, Andrés Cervantes ${ }^{2}$, Shanti Moreno Vera ${ }^{3}$ \& Julien Taieb 4 \\ ${ }^{1}$ Antwerp University Hospital, Edegem, Belgium \\ ${ }^{2}$ CIBERONC, Department of Medical Oncology, Biomedical Research institute INCLIVA, University of Valencia, Blasco Ibáñez 17, \\ 46010 Valencia, Spain \\ ${ }^{3}$ Global Medical Affairs, Oncology, Servier, Suresnes, France \\ ${ }^{4}$ Sorbonne Paris Cité, Hôpital Européen Georges Pompidou (HEGP), Paris - Descartes University, Assistance Publique-Hôpitaux de \\ Paris (AP-HP), 75015 Paris, France \\ *Author for correspondence: Tel.: +32 (3) 82143 66; Marc.Peeters@uza.be
}

Fluoropyrimidines are currently the backbone of treatment for gastrointestinal (GI) cancers but development of resistance to these agents remains a major problem. Trifluridine/tipiracil is an oral chemotherapeutic agent recently approved for third-line treatment of chemorefractory metastatic colorectal cancer. This article reviews the clinical value of trifluridine/tipiracil as a monotherapy, including recent trials in GI cancers, and the potential benefit of combining it with other agents in patients with GI cancers, including the preclinical rationale for combination therapy and recently completed and ongoing clinical trials. Data gathered so far suggest that trifluridine/tipiracil has the potential to form the chemotherapeutic backbone in the continuum of care for $\mathrm{Gl}$ cancers in the future.

First draft submitted: 22 February 2018; Accepted for publication: 4 April 2018; Published online: 27 April 2018

Keywords: colorectal cancer $\bullet$ gastrointestinal cancer $\bullet$ trifluridine/tipiracil

\section{Gastrointestinal cancers}

Gastrointestinal (GI) cancers, including colorectal cancer (CRC), gastric cancer (GC), esophageal cancer, gallbladder cancer, cancers of the liver, anal cancer and pancreatic cancer, are among the most frequently diagnosed malignancies worldwide and present a major public health burden [1].

CRC is the most common GI cancer in developed countries and one of the most common causes of cancerrelated deaths globally [2]. Approximately $25 \%$ of patients with CRC present with metastases at initial diagnosis, and $50 \%$ will go on to develop them [3]. Surgery may not be an option in metastatic patients and treatment is typically palliative with the aim of prolonging overall survival (OS) and maintaining or improving quality of life (QoL). The OS of patients with metastatic CRC (mCRC) has improved over the last two decades, with median OS reaching almost 30 months for selected groups of patients in some recent trials $[4,5]$. However, until recently, there were limited options for patients with disease progression through several lines of treatment.

GC also ranks among the leading causes of cancer mortality worldwide [1] and has particularly high incidence rates in eastern Asia, eastern Europe and South America [6]. Patients with advanced (locally advanced or metastatic) or recurrent GC have a poor prognosis, with a median OS of 10-12 months [7]. Hence, there remains a high unmet need for effective treatment options.

\section{Fluoropyrimidine-based treatment of Gl cancers}

The fluoropyrimidine, fluorouracil (5-FU), has remained one of the most extensively used chemotherapeutic agents for GI cancers since its introduction in 1957. Indeed, intravenously administered 5-FU and orally administered capecitabine or S-1, a prodrug of 5-FU, currently form the backbone of combination therapies for these cancers, and are recommended for first-line treatment of $\mathrm{mCRC}[8-10]$ and metastatic $\mathrm{GC}[6,11]$. The cytotoxic mechanism 
of action of 5-FU is primarily via the inhibition of thymidylate synthase (TS) by one of its metabolites, fluorodeoxyuridine monophosphate with $N^{5}, N^{10}$-methylenetetrahydrofolate, which impedes DNA synthesis [12]. Antitumor activity is also achieved through the misincorporation of 5-FU metabolites (fluoronucleotides) into DNA and RNA [13]. The response rate to intravenous 5-FU used alone as first-line treatment in CRC is, however, limited to $10-15 \%$ [14]. Strategies to improve the efficacy of 5-FU have included the addition of leucovorin (reduced folic acid) to enhance binding of fluorodeoxyuridine monophosphate to TS, and to use prodrug forms (e.g., capecitabine, which is preferentially converted to 5-FU in tumor tissue by thymidine phosphorylase [TP] [15]) or dihydropyrimidine dehydrogenase (DPD) inhibitors to limit 5-FU degradation by DPD in the liver [13]. Another viable strategy has been the addition of other chemotherapeutic agents to 5-FU-based regimens, such as oxaliplatin or irinotecan. These optimized regimens have lengthened the median OS in patients with previously untreated mCRC [16]. Nevertheless, resistance to 5-FU-based regimens, resulting from blocking of apoptosis, increased drug metabolism, drug target alterations and decreased ability to repair DNA damage, remains a major problem [14].

\section{Trifluridine/tipiracil}

Trifluridine/tipiracil (Lonsurf ${ }^{\circledR}$; also known as TAS-102) is an orally active agent composed of a thymidine-based nucleoside analog (trifluridine) and a TP inhibitor (tipiracil hydrochloride, hereafter referred to as tipiracil) at a molecular ratio of 1:0.5. Trifluridine/tipiracil was approved in the USA in September 2015 and the EU in April 2016 for patients with mCRC who have already been treated with, or cannot be given, other available treatments, including chemotherapy with fluoropyrimidines, oxaliplatin, irinotecan and anti-VEGF or, if $R A S$ wild-type, anti-EGF receptor (EGFR) monoclonal antibodies. In Japan, trifluridine/tipiracil was approved for unresectable advanced or recurrent mCRC in March 2014.

Trifluridine, an analog of thymidine, is the active cytotoxic component of trifluridine/tipiracil, with antitumor activity based primarily on its incorporation into DNA [12] as evidenced by the positive correlation between activity and the amount of trifluridine incorporated into DNA [17]. Following entry into cancerous cells via nucleoside transporters, trifluridine is phosphorylated by thymidine kinase to form trifluridine triphosphate, which is readily incorporated into DNA in place of thymidine bases [18]. This results in inhibition of cell proliferation and tumor growth. The incorporation of trifluridine triphosphate into DNA occurs with higher efficiency than 5-FU metabolites [19]. Trifluridine also inhibits TS, which is used in the synthesis of deoxythymidylate monophosphate for DNA synthesis [14], but this is believed to play a smaller role in its antitumor effects. Tipiracil is included in the formulation due to its TP-inhibiting properties. Trifluridine was originally synthesized in the 1960s, but clinical evaluation was discontinued at the time due to its poor pharmacokinetic (PK) profile and toxicity [20]. When administered alone, trifluridine is rapidly metabolized by TP in the liver and GI tract to inactive forms [21]. Adding tipiracil to the formulation thus increases the bioavailability of trifluridine, and hence its cytotoxicity, by inhibiting trifluridine degradation.

The differing primary mechanism of action of trifluridine to fluoropyrimidine (Figure 1) enables trifluridine/tipiracil to overcome acquired resistance to standard therapies. Indeed, trifluridine has demonstrated activity in 5-FU- and other fluoropyrimidine-resistant cell lines [12,22] and in fluoropyrimidine-refractory patients in clinical trials [23,24]. Trifluridine/tipiracil is considered to have a positive benefit-risk ratio based on trials in patients with heavily pretreated $\mathrm{mCRC}$, and is currently recommended by the main international guidelines, including European Society for Medical Oncology [9] and National Comprehensive Cancer Network consensus guidelines [8], as a third-line therapy for patients with refractory mCRC (level IB evidence).

Trifluridine/tipiracil has shown a consistent improvement in median OS (primary end point) over placebo in the late-line therapy of patients refractory to all available treatments, including fluoropyrimidines, in one Phase II and two Phase III randomized controlled trials (RECOURSE and TERRA). Consequently, there is a large amount of interest and ongoing research on the use of trifluridine/tipiracil in combination with other agents to further enhance the clinical benefit to patients. Possible combinations include trifluridine/tipiracil with other chemotherapeutic agents, signaling molecule inhibitors and immunotherapies. This article reviews the clinical value of trifluridine/tipiracil as a monotherapy and the potential benefits of trifluridine/tipiracil-based combinations with other agents in patients with GI cancers. 


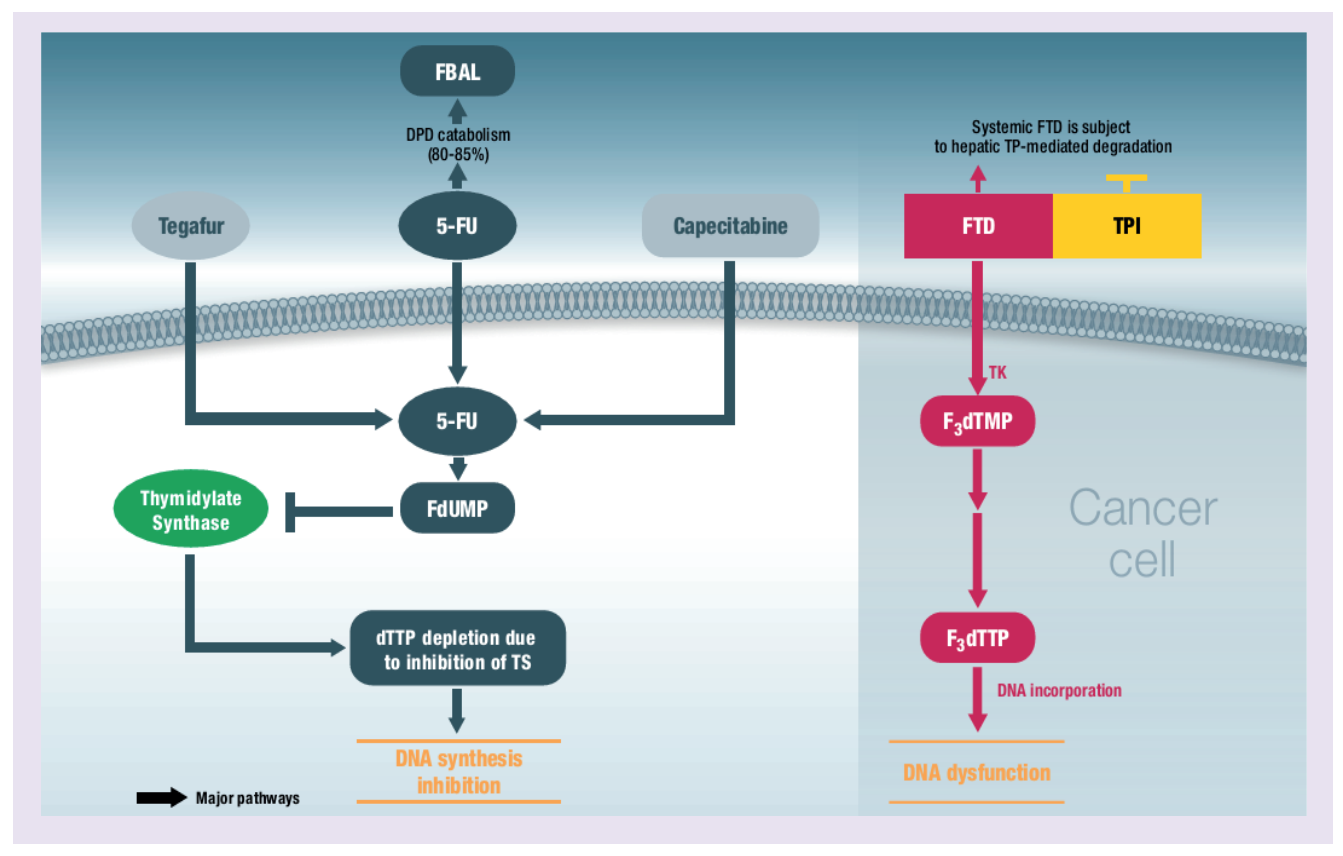

Figure 1. Comparison of the mechanisms of action of trifluridine/tipiracil (right-hand panel) versus fluorouracil (left-hand panel).

5-FU: Fluorouracil; DPD: Dihydropyrimidine dehydrogenase; dTTP: Deoxythymidine triphosphate; FBAL:

Alpha-fluoro-beta-alanine; $F_{3}$ dTMP: Trifluoromethyl deoxyuridine 5'-monophosphate; $F_{3}$ dTTP: Trifluoromethyl deoxyuridine 5'-triphosphate; FdUMP: Fluorodeoxyuridine monophosphate; FTD: Trifluorothymidine (trifluridine); TK: Thymidine kinase; TP: Thymidine phosphorylase; TPI: Tipiracil hydrochloride; TS: Thymidylate synthase.

Reproduced with permission from (c) Les Laboratoires Servier (2017).

\section{Clinical value of trifluridine/tipiracil monotherapy}

Efficacy of trifluridine/tipiracil in patients with $m C R C$

The therapeutic efficacy of trifluridine/tipiracil in the treatment of patients with mCRC, refractory or intolerant to standard therapies, was initially demonstrated in a Phase II trial in 169 Japanese patients [24] leading to registration in Japan, and results were confirmed in the pivotal Phase III trial (RECOURSE) in 800 patients [23] leading to worldwide registration.

The RECOURSE study was a randomized, double-blind, placebo-controlled, multinational trial to assess the efficacy and safety of trifluridine/tipiracil in patients with mCRC, refractory or intolerant to standard therapy [23]. Eligible patients had previously been treated with $\geq 2$ prior regimens of chemotherapy containing a fluoropyrimidine, oxaliplatin, irinotecan and bevacizumab, and an anti-EGFR therapy in patients with KRAS wild-type tumors. Patients were randomly assigned to receive twice-daily trifluridine/tipiracil $\left(35 \mathrm{mg} / \mathrm{m}^{2}\right)$ plus best supportive care (BSC) or placebo plus BSC and stratified by KRAS status, time between first diagnosis of metastases and randomization, and geographical region. Treatment was continued until disease progression, clinical progression, development of severe adverse events (AEs), withdrawal from the study or discontinuation of the patient by the investigator. The primary end point was OS. At the cutoff for primary analysis, 574 deaths had occurred and the median follow-up time was 11.8 months. At the cutoff for the updated survival analysis, 712 deaths had occurred [25]. Baseline characteristics were well balanced across the treatment groups. In the primary analysis, treatment with trifluridine/tipiracil plus BSC statistically and clinically significantly prolonged OS compared with placebo plus BSC (7.1 vs 5.3 months; hazard ratio [HR] for death 0.68; p < 0.001). These results were confirmed in the updated survival analysis (median OS: 7.2 vs 5.2 months; HR: 0.69). Median progression-free survival (PFS) was also statistically significantly prolonged in the trifluridine/tipiracil group compared with the placebo group (2.0 vs 1.7 months; HR for progression: 0.48; $\mathrm{p}<0.001$ ) at the date of primary analysis. The OS and PFS benefits were consistent across prespecified subgroups, including those with poorer prognosis at baseline (time since diagnosis of first metastases, Eastern Cooperative Oncology Group [ECOG] performance status [PS] and number of metastatic sites) and those with wild-type or mutated KRAS. A higher proportion of patients had disease control 
after 6 weeks in the trifluridine/tipiracil group than in the placebo group (44 vs 16\%), and trifluridine/tipiracil significantly delayed the worsening of ECOG PS from baseline compared with placebo ( $72 \%$ of patients in the trifluridine/tipiracil group and $81 \%$ in the placebo group had worsening of PS from 0 or 1 to $\geq 2$, with median time to worsening of 5.7 vs 4.0 months). The overall response rate (ORR) was $1.6 \%$ in the trifluridine/tipiracil group and $0.4 \%$ in the placebo group.

The efficacy and safety of trifluridine/tipiracil was further evaluated in a Phase III trial in an Asian population comprising 406 patients with mCRC, who had similar characteristics to the RECOURSE population [26]. To be enrolled, patients must have received at least two prior standard chemotherapy regimens for mCRC, including fluoropyrimidines, oxaliplatin and irinotecan, while prior anti-VEGF or anti-EGFR-targeted therapy was not mandatory. The efficacy results were consistent with those of RECOURSE: trifluridine/tipiracil plus BSC demonstrated a statistically significant improvement over placebo plus BSC for the primary end point of OS (median OS: 7.8 vs 7.1 months; HR: 0.79; $\mathrm{p}=0.035$ ) and the key secondary end point of PFS (median PFS: 2.0 vs 1.8 months; HR: 0.43; $\mathrm{p}<0.001)$.

The results of these clinical trials supported preclinical data indicating that the primary mechanism of action of trifluridine/tipiracil differs from that of fluoropyrimidine as efficacy was demonstrated in subgroups of patients who were refractory to fluoropyrimidines. In the subgroup of patients of RECOURSE who were refractory to treatment in which a fluoropyrimidine had been part of their last regimen (455 of 800 patients), superiority over placebo plus BSC was demonstrated for both OS (HR: 0.75) and PFS (HR: 0.51) [23].

\section{Safety profile \& QoL impact of trifluridine/tipiracil}

Trifluridine/tipiracil was found to have a good tolerability profile in patients with $\mathrm{mCRC}$ across trials, with AEs that were manageable by dose delay/reduction or supportive care [23,24]. The majority of AEs in patients treated with trifluridine/tipiracil in RECOURSE were hematological or GI [23]. Although the overall incidence of grade $\geq 3$ events was higher for trifluridine/tipiracil than for placebo, this was mostly accounted for by the hematological events, with other grade $\geq 3$ events occurring in less than $5 \%$ of patients. Among patients who took trifluridine/tipiracil, $38 \%$ had grade $\geq 3$ neutropenia and $4 \%$ had febrile neutropenia. Neutropenia was mostly managed successfully by reducing the dose, delaying the start of the next treatment cycle, and by use of granulocyte-colony stimulating factor, which was administered to $9 \%$ of patients. There was, however, one treatment-related death of a patient who took trifluridine/tipiracil, resulting from neutropenic infection-related septic shock. Common GI events in the trifluridine/tipiracil group included nausea ( $48 \%$, grade $\geq 3: 2 \%$ ), vomiting ( $28 \%$, grade $\geq 3: 2 \%)$, diarrhea $(32 \%$, grade $\geq 3: 3 \%)$ and abdominal pain $(21 \%$, grade $\geq 3: 2 \%)$. Other common AEs in the trifluridine/tipiracil group included decreased appetite (39\%, grade $\geq 3: 4 \%)$, fever (19\%, grade $\geq 3$ : $1 \%)$ and asthenia $(18 \%$, grade $\geq 3: 3 \%)$, all of which are manageable. Low frequencies of stomatitis $(8 \%$, grade $\geq 3$ : $<1 \%)$, hand-foot syndrome ( $2 \%$, no grade $\geq 3$ events) and cardiac events $(<1 \%$, grade $\geq 3:<1 \%)$ were reported in the trifluridine/tipiracil group, with no clinically relevant differences in these frequencies between treatment groups. The good tolerability profile of trifluridine/tipiracil overall was emphasized by the low rate of treatment discontinuation following AEs (4\%) and the low proportion of patients requiring dose reductions (14\%).

Analysis of QoL proxies in the RECOURSE population, namely the relationship between AEs and discontinuations, and ECOG PS, suggested that trifluridine/tipiracil did not result in deterioration of QoL versus placebo [27]. While AEs affecting QoL occurred more frequently and with longer duration in the trifluridine/tipiracil group than the placebo group, all AEs (except nausea and vomiting) occupied a smaller proportion of the total treatment period. Moreover, among patients who had their PS recorded at discontinuation $(\mathrm{n}=759)$, PS was maintained in $67 \%$ in the trifluridine/tipiracil group and $63 \%$ in the placebo group; $84 \%$ in the trifluridine/tipiracil group and $81 \%$ in the placebo group remained at PS 0 or 1 at discontinuation. In addition, a post hoc analysis of Qualityadjusted Time Without Symptoms of disease or Toxicity was conducted by partitioning OS into three discrete health states (toxicity, time without symptoms or toxicity and relapse) and combining the mean duration of each state, weighted by a utility coefficient representing QoL, into a global quality-adjusted time without symptoms of disease or toxicity score [28]. The results favored trifluridine/tipiracil, with a clinically meaningful improvement in quality-adjusted survival of 1.5 months over placebo (5.48 vs 3.98 months). This indicated that most of the OS gain in the RECOURSE trial was spent in a health state (1.5 of 1.8 months survival difference compared with placebo) where patients were not experiencing grade $3 / 4$ AEs. Overall, the maintenance of PS and improvement in quality survival time in patients given trifluridine/tipiracil, as indicated by these data, is an important contributing factor to the clinical value of trifluridine/tipiracil as a later-line treatment for mCRC. 


\section{Efficacy \& safety of trifluridine/ tipiracil compared with other treatments for $m C R C$}

The toxicity profile of trifluridine/tipiracil differs from that of fluoropyrimidine. Indeed, AEs associated with fluoropyrimidine use (febrile neutropenia, stomatitis, hand-foot syndrome and cardiac ischemia) were found in few patients who took trifluridine/tipiracil in the RECOURSE trial (grade $\geq 3:<1 \%$ for all but febrile neutropenia [4\%]). In contrast to 5-FU, trifluridine/tipiracil can be given to patients with DPD deficiency, as TP not DPD is the primary enzyme that metabolizes trifluridine [18,21].

Safety/tolerability and QoL impact are important determinants of the clinical value of treatments in late-stage GI cancers. Regorafenib, a multitargeted kinase inhibitor, is the only other agent approved for third-line treatment of patients with refractory mCRC [9]. This agent has comparable efficacy to trifluridine/tipiracil, with a survival improvement of a similar magnitude, but a differing toxicity profile [29]. Hand-foot syndrome and increased liver enzymes were more frequent with regorafenib, while neutropenia, leukopenia, anemia, nausea and febrile neutropenia were more frequent with trifluridine/tipiracil [29]. Trifluridine/tipiracil was found to score higher than regorafenib in a recent grading of overall clinical benefit of the US FDA-approved new drugs for treating advanced solid cancer (2000-2015), both by American Society of Clinical Oncology Value Framework and European Society for Medical Oncology Magnitude of Clinical Benefit Scale scoring systems, which consider treatment effect, toxicity and QoL [30].

\section{Ongoing research on trifluridine/tipiracil monotherapy}

Clinical research is underway to investigate the use of trifluridine/tipiracil as a treatment in GC, for which there remains an unmet need for treatment options in patients with locally advanced or metastatic disease [7,31].

A multicenter, single-arm, Phase II study has been conducted in Japan evaluating the safety and efficacy of trifluridine/tipiracil ( $35 \mathrm{mg} / \mathrm{m}^{2}$ twice daily) in 29 patients with advanced GC who had failed standard chemotherapies, including fluoropyrimidines, platinum and any of the taxanes or irinotecan (UMIN000007421 [32]). Trifluridine/tipiracil was well tolerated in these patients and there were only two discontinuations due to AEs, and no treatment-related deaths. Common grade $\geq 3$ AEs included neutropenia (69\%), leukopenia (41.4\%), anemia $(20.7 \%)$ and anorexia (10.3\%). The efficacy results were positive, with a clinically relevant investigator-determined disease control rate (DCR) of $65.5 \%$, and an independent central review-determined DCR of 51.9\%, after 8 weeks of treatment. In addition, investigator-determined median PFS was 2.9 months, and at a median follow-up time of 17.1 months, investigator-determined median OS was 8.7 months.

Based on the encouraging Phase II data, the efficacy and safety of trifluridine/tipiracil plus BSC compared with placebo plus BSC is currently being evaluated in a global, randomized, double-blind Phase III trial in an expected 500 patients with metastatic GC refractory to standard treatments (TAGS study; NCT02500043 [33]). The primary end point is OS and secondary end points include PFS, QoL and safety assessments. The results are expected in 2018.

\section{Combinations with trifluridine/tipiracil}

The introduction of combination regimens of different chemotherapeutic agents (e.g., 5-FU, leucovorin and oxaliplatin [FOLFOX]) and/or molecularly targeted agents has lengthened survival time in patients with mCRC compared with earlier treatment with 5-FU alone [34]. Combining trifluridine/tipiracil with other anticancer agents, therefore, has the potential to enhance its efficacy. Intensive research is now underway to evaluate trifluridine/tipiracil-based combination regimens with chemotherapies (e.g., oxaliplatin and irinotecan), targeted therapies (e.g., VEGF and EGFR inhibitors) and immunotherapies (e.g., PD-1/PD-L1 inhibitors; Figure 2). Preclinical data have supported the rationale for clinical use of such combinations and key data are summarized below. The clinical trials that have been completed, are ongoing or planned for these combinations are presented in Table 1 and discussed below.

\section{Trifluridine/ tipiracil with other chemotherapeutic agents}

With irinotecan

Irinotecan $\left(\right.$ Camptostar ${ }^{\circledR}$ ) inhibits topoisomerase I causing DNA damage and subsequent cell death. This agent is principally used in combination with 5-FU, leucovorin and oxaliplatin (FOLFOXIRI or FOLFIRI) for treatment of CRC, but may also be used alone. Preclinical evaluation of the antitumor activity of a number of cytotoxic agents in combination with trifluridine/tipiracil found the combination of trifluridine/tipiracil and irinotecan to be the most promising among those tested. Trifluridine/tipiracil with irinotecan showed superior inhibition of 


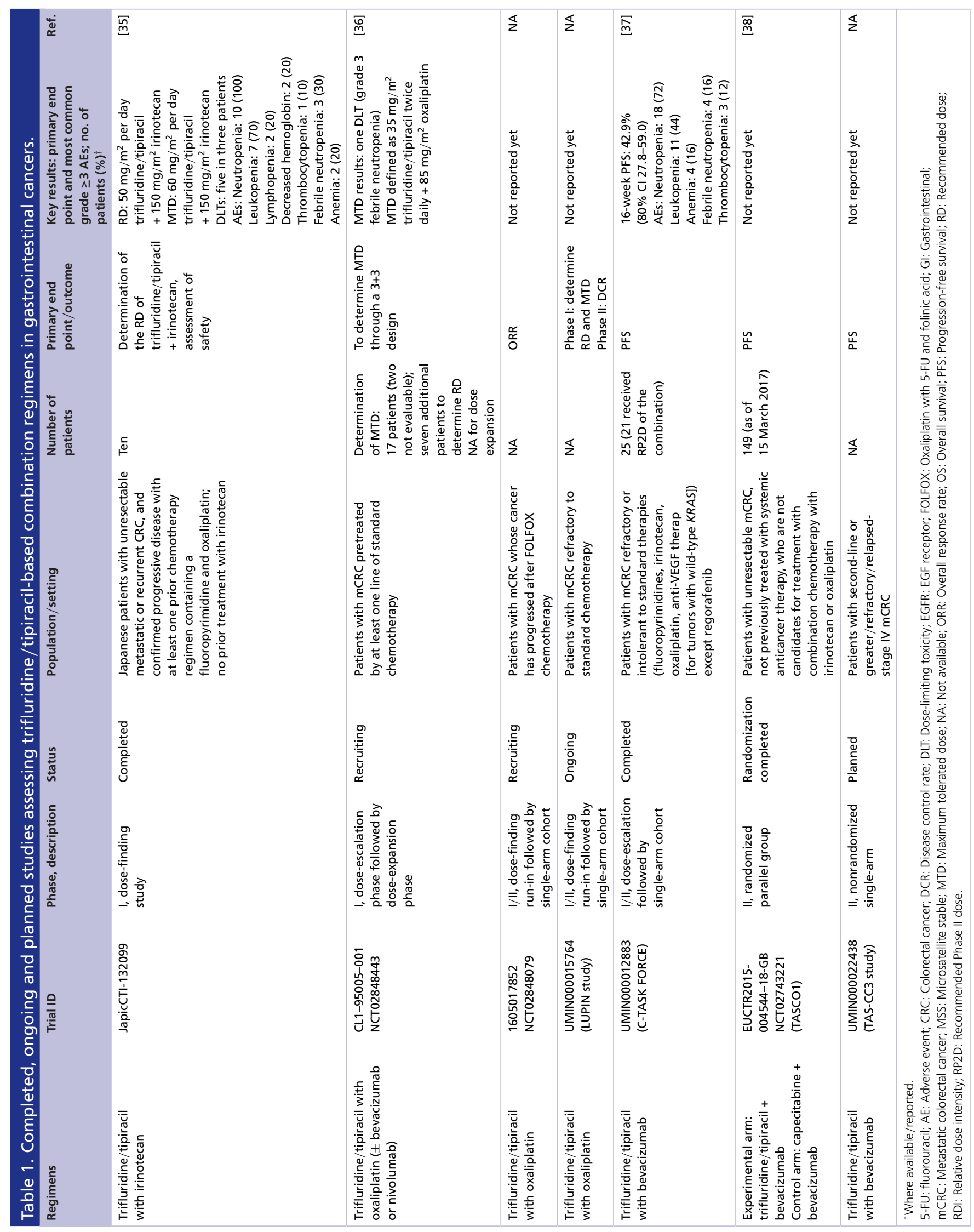




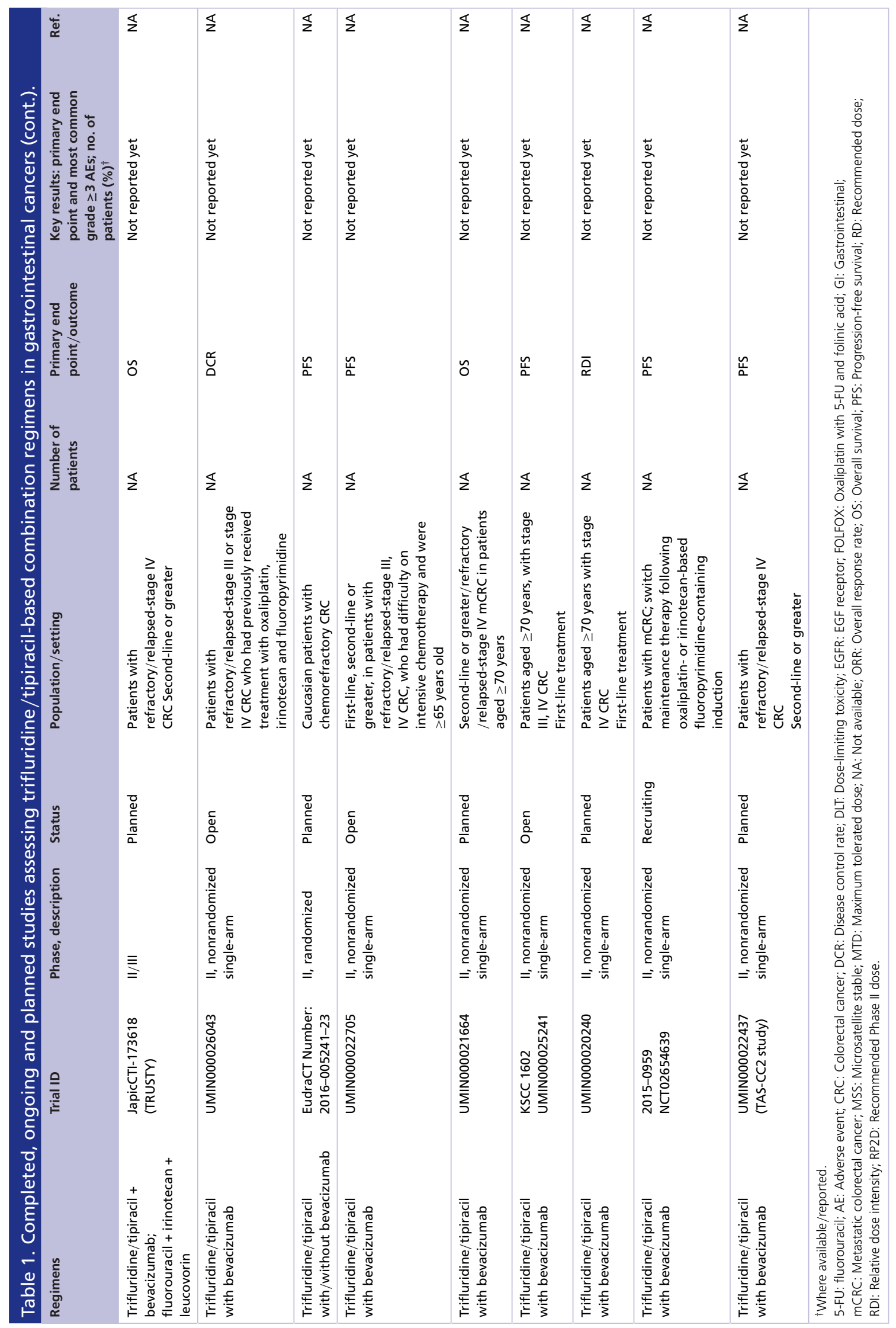


Review Peeters, Cervantes, Moreno Vera \& Taieb

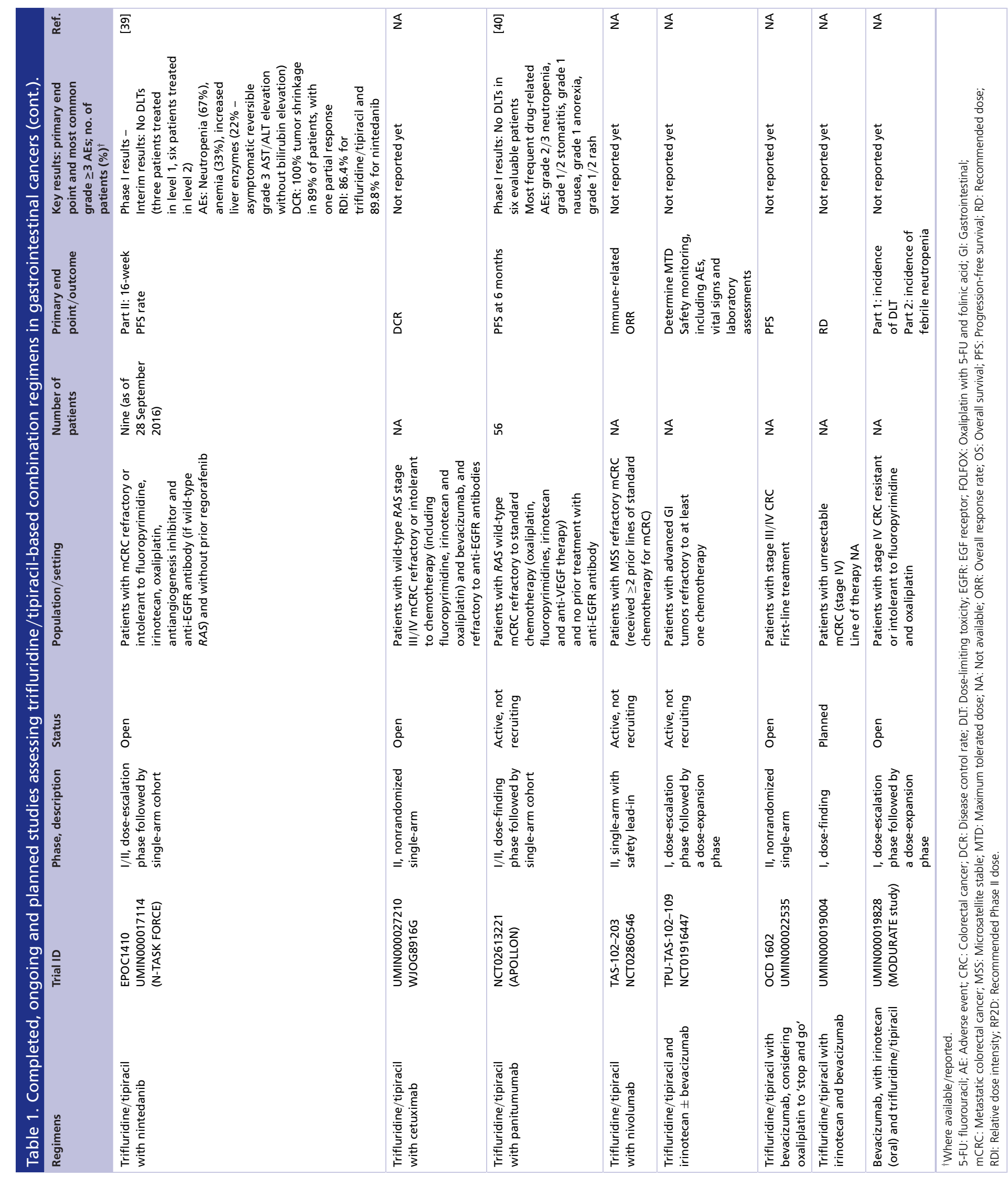




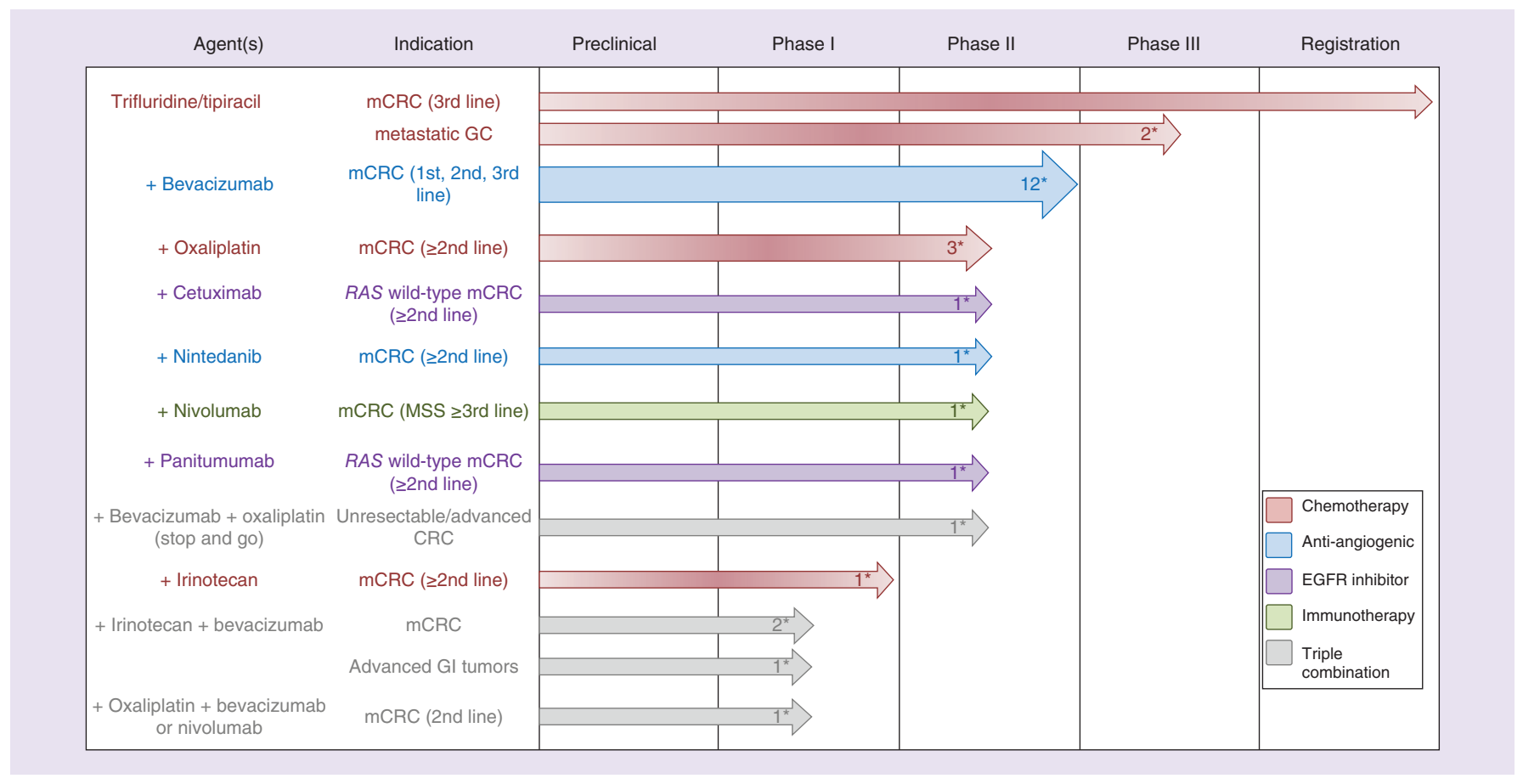

Figure 2. Overview of clinical development pipeline of trifluridine/tipiracil-containing combination regimens.

* Numbers refer to the number of known completed, ongoing or planned clinical trials for the combination in that indication. CRC: Colorectal cancer; EGFR: EGF receptor; GC: Gastric cancer; GI: Gastrointestinal; mCRC: Metastatic colorectal cancer; MSS: Microsatellite stable.

tumor growth compared with either agent alone in a CRC and GC xenograft-bearing nude mouse model, and was active in 5-FU-resistant cell lines [41]. Furthermore, SN38, an active metabolite of irinotecan, exhibited enhanced cytotoxicity in colon cancer cells in vitro when preincubated with trifluridine [42].

The positive preclinical data led to the design of a Phase I trial to determine the recommended dose (RD) and assess safety of trifluridine/tipiracil with irinotecan in Japanese patients with mCRC refractory to 5-FU and oxaliplatin (JapicCTI-132099 [35]). Enrolled patients were administered escalating doses of trifluridine/tipiracil twice daily $\left(40-70 \mathrm{mg} / \mathrm{m}^{2}\right.$ per day), and irinotecan by intravenous infusion at a fixed dose of $150 \mathrm{mg} / \mathrm{m}^{2} \mathrm{for} \mathrm{a}$ minimum of $90 \mathrm{~min}$ on days 1 and 15 of each 28-day schedule. A total of ten patients were enrolled, with one excluded from the analysis of dose-limiting toxicities (DLTs) and efficacy. There were five DLTs in three patients: one patient with a grade 3 febrile neutropenia and grade 4 neutropenia at $50 \mathrm{mg} / \mathrm{m}^{2}$ per day, and two patients, both with grade 3 febrile neutropenia and one with grade 4 neutropenia, at $60 \mathrm{mg} / \mathrm{m}^{2}$ per day. The combination $\mathrm{RD}$ was thus determined to be $50 \mathrm{mg} / \mathrm{m}^{2}$ per day of trifluridine/tipiracil with $150 \mathrm{mg} / \mathrm{m}^{2}$ of irinotecan, although it was considered that further optimization of the regimen would be needed, given the low relative dose intensity of irinotecan: $32.6 \%$ at $60 \mathrm{mg} / \mathrm{m}^{2}$ per day of trifluridine/tipiracil dose escalation. The most common grade $\geq 3$ treatment-related AEs were neutropenia (100\%), leukopenia (70\%), febrile neutropenia (30\%), lymphopenia $(20 \%)$ and anemia (20\%). Neutropenia, leukopenia and febrile neutropenia all occurred at a higher frequency in this study than seen with trifluridine/tipiracil monotherapy or other regimens with irinotecan [35]. However, these toxicities were controlled using appropriate measures. The efficacy results for the combination were encouraging, with partial responses achieved in $22 \%$ of the patients, and a DCR of $55.6 \%$.

\section{With oxaliplatin}

Oxaliplatin is a platinum derivative, which primarily exerts its cytotoxic effect through induction of DNA strand breaks [43]. The antitumor activity of oxaliplatin alone is modest and it is therefore usually administered in combination with other agents, such as fluoropyrimidines. Preclinical studies revealed oxaliplatin to be a promising combination partner for trifluridine/tipiracil in the treatment of CRC or GC. An in vitro study demonstrated synergy between these agents, for both fixed and variable agent ratios, in terms of DNA damage and apoptosis in CRC cell lines [44]. Oxaliplatin-induced DNA damage and apoptosis were enhanced by the presence of trifluridine, 
reportedly due to increased formation of Pt-DNA adducts resulting from increased incorporation of trifluridine into DNA. The combination also showed superior inhibition of tumor growth compared with either agent alone in a CRC and GC xenograft-bearing nude mouse model, and importantly, was active in 5-FU-resistant gastric tumors [45].

A Phase I dose-escalation study of trifluridine/tipiracil in combination with oxaliplatin in patients with $\mathrm{mCRC}$ previously treated with $\geq 1$ line of standard chemotherapy is ongoing (NCT02848443 [36]). The trial includes a doseescalation phase with a $3+3$ design to determine the maximum tolerated dose (MTD) and RD of the combination, and an expansion phase to explore its safety, PK and preliminary efficacy with bevacizumab or nivolumab. To avoid overlapping toxicity of trifluridine/tipiracil, particularly with regards to neutropenia, trifluridine/tipiracil is being administered with a 14-day schedule (twice-daily for 5 days followed by 9 days of recovery at doses of 25, 30 and $35 \mathrm{mg} / \mathrm{m}^{2}$ ) rather than the standard 28-day cycle. Oxaliplatin is being administered intravenously on day 1 of each treatment cycle, every 2 weeks, at a dose of $85 \mathrm{mg} / \mathrm{m}^{2}$, with the possibility of de-escalating to $65 \mathrm{mg} / \mathrm{m}^{2}$ in the event of DLTs. In 17 patients enrolled for dose escalation (two not evaluable), one DLT of grade 3 febrile neutropenia was reported at the dose of $35 \mathrm{mg} / \mathrm{m}^{2}$ trifluridine/tipiracil plus $85 \mathrm{mg} / \mathrm{m}^{2}$; the DLT was resolved and the patient continued with a dose reduction to $30 \mathrm{mg} / \mathrm{m}^{2}$ of trifluridine/tipiracil. The MTD was defined as the maximal planned dose of $35 \mathrm{mg} / \mathrm{m}^{2}$ trifluridine/tipiracil twice daily plus $85 \mathrm{mg} / \mathrm{m}^{2}$ oxaliplatin. Preliminary efficacy was promising, with one unconfirmed partial response and seven cases of stable disease reported.

A Phase I/II trial of the combination in patients with $\mathrm{mCRC}$ that progressed after $\geq 2$ lines of standard therapy including 5-FU, irinotecan and oxaliplatin has also begun recruitment (NCT02848079). An estimated 68 patients are to be enrolled and the primary outcome is the ORR as assessed by RECIST v1.1. Similarly, a Phase I/II study of trifluridine/tipiracil combined with oxaliplatin in mCRC patients refractory to standard chemotherapy is ongoing in Japan (the LUPIN study; UMIN000015764).

\section{With docetaxel}

Docetaxel (Taxotere ${ }^{\circledR}$ ) is a taxane, which exerts its cytotoxic activity by microtubule inhibition [46]. It is considered a standard chemotherapeutic agent for the treatment of a number of different types of solid tumors. A preclinical study investigated sequential and concurrent combinations of trifluridine with docetaxel in human CRC cell lines [46]. The combination showed synergistic activity, with administration of docetaxel followed by trifluridine producing the most potent induction of cell death, polynucleation and mitotic spindle inhibition. This was considered most likely related to the combined induction of cell death and mitotic failure. While these data provide strong rationale for clinical use of the combination, there have been no clinical trials to date.

\section{Trifluridine/tipiracil with targeted therapies}

With bevacizumab

Fluoropyrimidine-based combination regimens with bevacizumab (Avastin ${ }^{\circledR}$ ), a monoclonal antibody that blocks angiogenesis by targeting VEGF [47], are used as first-line treatments for mCRC due to good efficacy and a lack of overlapping toxicity [23,48]. Preclinical studies have evaluated the antitumor synergy of trifluridine/tipiracil and bevacizumab, with positive results: in CRC xenografts, inhibition of tumor growth was significantly enhanced with trifluridine/tipiracil plus bevacizumab compared with either agent alone [49]. In addition, levels of phosphorylated trifluridine were increased when combined with bevacizumab, suggesting that bevacizumab may help increase trifluridine accumulation and its subsequent phosphorylation in tumors by normalizing tumor vasculature. This provided a strong rationale with which to proceed to clinical evaluation of this combination.

The efficacy and safety of trifluridine/tipiracil plus bevacizumab has been evaluated in a multicenter Phase I/II trial (C-TASK FORCE; UMIN000012883) in patients with mCRC who were refractory or intolerant to standard therapies (fluoropyrimidines, irinotecan, oxaliplatin, anti-VEGF therapy [for tumors with wild-type KRAS]) except regorafenib [37]. The trial included a Phase I dose de-escalation in which the recommended Phase II dose (RP2D) was determined and a Phase II expansion in which patients received the RP2D. The primary end point was centrally assessed PFS at 16 weeks, analyzed in the first 21 patients enrolled and treated with the RP2D. A threshold of nine of 21 patients (42.9\%) without progression at 16 weeks was set for the primary objective to be achieved, based on Phase II trifluridine/tipiracil monotherapy data [24]. In Phase I, six patients were enrolled and received $35 \mathrm{mg} / \mathrm{m}^{2}$ trifluridine/tipiracil twice daily plus bevacizumab $(5 \mathrm{mg} / \mathrm{kg}$ intravenously for 30 min every 2 weeks) at level 1 of dose de-escalation. There were no reported DLTs at this dose and it was defined as the RP2D. Of 21 patients in the primary analysis set who received the RP2D, nine (42.9\%) had no centrally assessed disease 
progression at 16 weeks; the primary objective was therefore met. The median PFS was 3.7 months and, after 22 deaths, the median OS was 11.4 months. The centrally assessed DCR was 64\%. Together, these data indicated promising antitumor activity, albeit in a small population. The combination had acceptable toxicity and, as with trifluridine/tipiracil monotherapy, hematological events were the most commonly reported grade 3 AEs. These events were manageable with dose reduction, temporary interruption and/or granulocyte-colony stimulating factor. There were no treatment-related deaths and no treatment discontinuations due to drug-related toxicity. Relative dose intensities were similar to that seen for trifluridine/tipiracil monotherapy.

Several Phase II trials are now underway and planned to evaluate the combination in first-line therapy of subpopulations of patients not eligible for intensive treatment (TASCO1; EUCTR2015-004544-18-GB), in elderly patients ( $\geq 70$ years old; UMIN000025241, UMIN000020240, UMIN000022705), as second- (JapicCTI173618 and UMIN000022437) and third-line therapy (UMIN000022438 and EudraCT: 2016-005241-23, UMIN000021664), as therapy in patients who had previously received oxaliplatin, irinotecan and fluoropyrimidine (UMIN000026043), and in the maintenance setting after standard first-line induction (NCT02654639 [50]).

The TASCO1 study is an ongoing, international, randomized Phase II trial evaluating trifluridine/tipiracil with bevacizumab and capecitabine with bevacizumab in patients with unresectable mCRC (diagnosed within 6 months before first drug administration) who are noneligible for intensive oxaliplatin- or irinotecan-based chemotherapy [38]. The 149 enrolled patients have been stratified by RAS status, ECOG PS and country. The primary end point is PFS, which will be performed when 100 PFS events have been recorded. Other assessments include ORR, duration of response, DCR, OS, safety and QoL questionnaires.

With nintedanib

Nintedanib $\left(\mathrm{Ofev}^{\circledR}\right.$ and Vargatef $\left.{ }^{\circledR}\right)$ is a small-molecule triple angiokinase inhibitor of VEGF, PDGF and FGF receptors, approved in non-small-cell lung carcinoma and idiopathic pulmonary fibrosis. A global Phase III trial (LUME-colon 1) of nintedanib monotherapy in patients with mCRC refractory to all available treatments, showed improved PFS, but not OS, versus placebo. The rationale for combining trifluridine/tipiracil and nintedanib is based on preclinical data demonstrating enhanced antitumor activity and increased concentrations of trifluridine incorporated into DNA in CRC xenografts in nude mice, compared with either agent alone [51], and the convenience of having two oral drugs. An ongoing multicenter Phase I/II study (EPOC1410; N-TASK FORCE) has been designed to evaluate the efficacy and safety of the combination in patients with mCRC refractory or intolerant to standard therapies (fluoropyrimidines, irinotecan, oxaliplatin, antiangiogenesis inhibitors, anti-EGFR antibody [if wild-type $R A S$ ]) and without prior regorafenib, with results available for Phase I [39]. A $3+3$ cohort-based dose escalation design of nintedanib (150 and $200 \mathrm{mg}$ twice daily every day on levels 1 and 2, respectively) added to the standard dose of trifluridine/tipiracil was used to determine the RP2D in Phase I. The regimen had acceptable tolerability at the doses tested. No DLTs were observed in the three patients treated at level 1 or six patients at level 2; the RP2D was defined as $200 \mathrm{mg}$ twice daily of nintedanib. The most common grade $\geq 3$ AEs were neutropenia (67\%), anemia (33\%) and increased liver enzymes (22\% with reversible grade 3 AST/ALT elevation without bilirubin elevation). The combination showed promising antitumor activity in Phase I, with a DCR of $100 \%$ and evidence of tumor shrinkage in $89 \%$ of patients (including one partial response).

With EGFR inhibitors (cetuximab, panitumumab \& erlotinib)

Cetuximab (Erbitux ${ }^{\circledR}$ ) and panitumumab (Vectibix ${ }^{\circledR}$ ) are monoclonal antibodies targeting EGFR, which is overexpressed in many GI cancers [52]. These agents have been approved in the EU and USA for previously untreated RAS wild-type patients with $\mathrm{mCRC}$ in combination regimens with 5-FU, irinotecan or oxaliplatin, or as monotherapies for patients who failed, or are intolerant to, standard chemotherapies. A preclinical study has shown that combining cetuximab or panitumumab with trifluridine/tipiracil results in promising antitumor activity in KRAS wild-type CRC xenografts, which was enhanced compared with these agents alone [53].

Based on these positive results, an ongoing Phase I/II study (APOLLON; NCT02613221) has been designed to investigate the efficacy and safety of panitumumab with trifluridine/tipiracil in patients with $R A S$ wild-type mCRC refractory or intolerant to standard chemotherapies (fluoropyrimidines, irinotecan, oxaliplatin) and antiangiogenics, who had no prior treatment with anti-EGFR antibodies, trifluridine/tipiracil or regorafenib [40]. Results for the Phase I 3+3 dose de-escalation (panitumumab $6 \mathrm{mg} / \mathrm{kg}$ by intravenous infusion on days 1 and 15 every 4 weeks per course; trifluridine/tipiracil $35 \mathrm{mg} / \mathrm{m}^{2}$ twice daily on days $1-5$ and $8-12$, every 4 weeks) to define the RP2D have been reported. Among six evaluable patients, there were no reported DLTs and the RP2D was thus set as 
the standard doses of panitumumab and trifluridine/tipiracil. Three patients achieved a partial response and three had stable disease. Further efficacy data will be provided by Phase II, which is ongoing and will assess the primary end point: the rate of PFS at 6 months by investigator assessment, in a planned 46 patients. The combination of trifluridine/tipiracil and cetuximab is also being evaluated in a Phase II trial in Japan in patients with $R A S$ wild-type mCRC refractory to standard chemotherapies and to previous anti-EGFR antibodies (UMIN000027210). Patients are to be treated at the standard dose of trifluridine/tipiracil and at an initial dose of $400 \mathrm{mg} / \mathrm{m}^{2}$ of cetuximab followed by weekly doses of $250 \mathrm{mg} / \mathrm{m}^{2}$.

Erlotinib (Tarceva ${ }^{\circledR}$ ) is an orally active reversible inhibitor of the tyrosine kinase domain of EGFR that is currently used in treatment of advanced or metastatic non-small-cell lung cancer and metastatic pancreatic cancer (in combination with gemcitabine). There is potential to use erlotinib in CRC, with anticancer activity previously shown in patients with mCRC [54], particularly in combination with chemotherapy. A preclinical study showed that trifluridine and erlotinib were synergistic in most CRC cell lines tested, including wild-type EGFR-expressing cells and those with KRAS mutations [55]. However, the combination was not synergistic in cells that did not express EGFR. The synergy was considered to be related to induction of cell cycle arrest and erlotinib-mediated inhibition of prosurvival signaling that was activated through phosphorylation by trifluridine. These synergistic molecular mechanisms present a sound rationale for combining trifluridine/tipiracil with EGFR inhibitors for treatment of CRC.

\section{Trifluridine/tipiracil with immunotherapies}

With PD-1/PD-L1 inhibitors

Antibodies targeting the immune checkpoint inhibitor receptor, PD-1, and its ligand, PD-L1, have shown durable responses and acceptable safety in several tumors. In CRC, PD-1/PD-L1 inhibitor monotherapies have the most potential in patients whose tumors have mismatch repair proteins with microsatellite instability, as this subset of patients exhibit higher responses than microsatellite-stable (MSS) patients [56].

Combining anti-PD-1 antibodies with chemotherapy has the potential to be more effective than anti-PD-1 monotherapy, particularly in patients with MSS disease for whom no benefits have yet been seen with anti-PD1/PD-L1 monotherapy but who account for the majority of patients with mCRC [57]. This is, in part, due to the ability of some chemotherapeutic agents to cause immunogenic cell death (ICD), thereby eliciting an anticancer immune response. To this end, the immunogenic properties of oxaliplatin and trifluridine/tipiracil have been evaluated in vitro, and the therapeutic effect of the triple combination of oxaliplatin and trifluridine/tipiracil with anti-PD-1 in vivo, in murine MSS CT26 colon carcinoma [58]. Trifluridine/tipiracil and oxaliplatin in combination were found to increase ICD in vitro, as indicated by a panel of ICD markers, compared with either agent alone. Moreover, in vivo, the triple combination led to significantly increased survival of mice as compared with trifluridine/tipiracil with oxaliplatin or trifluridine/tipiracil with anti-PD-1, and modest tumor growth inhibition. In another study in mice, treatment of MSS CRC tumors with trifluridine/tipiracil and an anti-PD-1 antibody resulted in statistically significantly greater tumor growth inhibition compared with either monotherapy, and led to complete tumor regression in four/five mice [59]. A Phase II, multicenter, single-arm, safety lead-in trial to assess safety and efficacy of trifluridine/tipiracil with nivolumab (Opdivo $\left.{ }^{\circledR}\right)$, a PD-1 antibody, in patients with MSS refractory mCRC is currently active (NCT02860546). The primary outcome is the immune-related ORR, defined as the incidence of complete and partial responses in the efficacy population.

\section{Triple combinations with trifluridine/tipiracil}

Research is also underway to investigate the potential clinical use of triple combination regimens with trifluridine/tipiracil. A two-part Phase I study (NCT01916447) is ongoing and aims to determine the MTD and evaluate safety, PK and preliminary efficacy of trifluridine/tipiracil plus irinotecan, with or without bevacizumab, in an estimated 65 patients with advanced GI tumors refractory to at least one chemotherapy. In addition, Phase I trials of trifluridine/tipiracil with irinotecan and bevacizumab are in progress in patients with unresectable mCRC (UMIN000019004) and with mCRC refractory to fluoropyrimidine and oxaliplatin (MODURATE study; UMIN000019828). The combination of trifluridine/tipiracil and bevacizumab, with oxaliplatin given in a 'stopand-go' manner, is being assessed for first-line treatment of patients with advanced unresectable CRC in a Phase II study (UMIN000022535), while trifluridine/tipiracil with oxaliplatin, and bevacizumab or nivolumab, is being evaluated as second-line treatment in patients with mCRC in the expansion phase of the NCT02848443 study [36]. 


\section{Discussion \& future perspective}

Trifluridine/tipiracil is a relatively new agent in oncology that has so far been approved for third-line treatment of patients with refractory mCRC. However, ongoing research is providing evidence of its benefit, both as a monotherapy and as a component or backbone of combination regimens, in earlier lines of treatment of CRC and in other GI cancers, such as metastatic GC. The current clinical program is highly active, with numerous trifluridine/tipiracil-based combinations undergoing evaluation in clinical trials following preclinical data supporting the rationale for their clinical use.

The differing primary mechanism of action of trifluridine/tipiracil to fluoropyrimidine has enabled its successful use as a monotherapy in patients with fluoropyrimidine-refractory mCRC who are known to be very difficult to treat. Trifluridine/tipiracil has shown a consistent improvement in median OS in patients with refractory mCRC by approximately 2 months compared with placebo [25], which is similar to that seen with regorafenib - the only other agent approved for third-line treatment of mCRC. However, the tolerability of trifluridine/tipiracil differs from regorafenib, with hematological events accounting for the majority of grade $\geq 3$ events in patients who took trifluridine/tipiracil in the pivotal trial, and the frequencies of hand-foot syndrome and hepatic toxicities being much lower than those seen with regorafenib [29]. Owing to the differing mechanisms of action of trifluridine/tipiracil and regorafenib, sequential treatment may be beneficial in patients with refractory mCRC. However, there are currently insufficient data assessing sequential use of these agents to provide a consensus on this approach $[60,61]$. Importantly, especially in the third-line treatment setting, trifluridine/tipiracil maintained PS in patients during the pivotal trial, as indicated by analyses of QoL proxies, suggesting that it may be better to use it first and maintain a good PS for as long as possible. As a monotherapy, trifluridine/tipiracil also has a potential application as a treatment in patients with metastatic GC who have failed standard chemotherapies, with promising results shown in the recent Phase II trial [32], and results of the Phase III trial expected in 2018 [33].

Clinical trials of several combination regimens with trifluridine/tipiracil are underway and positive Phase I/II preliminary efficacy data have already been obtained for trifluridine/tipiracil in combination with the chemotherapy, irinotecan and oxaliplatin; the VEGF inhibitor, bevacizumab; the angiokinase inhibitor, nintedanib; and the EGFR inhibitor, panitumumab. Data from the C-TASK FORCE study evaluating trifluridine/tipiracil with bevacizumab [37] are particularly promising with $9 / 21$ (43\%) patients who received the combination showing no progression at 16 weeks, exceeding the prespecified threshold to meet the primary objective based on trifluridine/tipiracil monotherapy data. Limitations of the study, however, included the relatively small sample size and the exclusion of patients previously treated with regorafenib, which may not accurately represent the real clinical situation in the refractory mCRC setting [62]. This combination is now being evaluated in other Phase II trials in mCRC in the first-line setting in patients ineligible for intensive treatment, elderly patients ( $\geq 70$ years of age), the second-line setting and maintenance setting after standard induction.

Overall, the current development plan for trifluridine/tipiracil is very promising. Preclinical and clinical data gathered from studies of trifluridine-/tipiracil-based combinations so far suggest that this agent has the potential to form the chemotherapeutic backbone in the continuum of care for GI cancers in the future.

\section{Supplementary data}

A video abstract and transcript are available as an accompaniment to this paper. To view the supplementary transcript for this, please visit the journal website at: www.futuremedicine.com/doi/suppl/10.2217/fon-2018-0147

\section{Acknowledgements}

Assistance with drafting of the manuscript was provided by J Kistler, Scinopsis (Fréjus, France), funded by Servier.

\section{Financial \& competing interests disclosure}

$\mathrm{M}$ Peeters has received research grants and/or honoraria from Taiho and/or Servier. A Cervantes has received research grants and/or honoraria from Taiho and/or Servier. S Moreno Vera is an employee of Servier. J Taieb has received honoraria from Servier, Amgen, Roche-Genentech, Merck Serono, Elli Lilly, Celgene, Sirtex, Shire and Sanofi. The authors have no other relevant affiliations or financial involvement with any organization or entity with a financial interest in or financial conflict with the subject matter or materials discussed in the manuscript apart from those disclosed. 
Open access

This work is licensed under the Attribution-NonCommercial-NoDerivatives 4.0 Unported License. To view a copy of this license, visit http://creativecommons.org/licenses/by-nc-nd/4.0/

Executive summary

- Fluoropyrimidines are currently the backbone of treatment for gastrointestinal (GI) cancers but development of resistance to these agents remains a major problem.

- Trifluridine/tipiracil is an orally active chemotherapeutic agent recently approved for third-line treatment of chemorefractory metastatic colorectal cancer (mCRC).

- The primary mechanism of action of trifluridine/tipiracil is via the incorporation of phosphorylated trifluridine into DNA, in contrast to fluoropyrimidine which primarily inhibits thymidylate synthase. Tipiracil is included in the formulation to increase the bioavailability of trifluridine.

- The differing mechanism of action of trifluridine/tipiracil to fluoropyrimidine enables it to overcome fluoropyrimidine resistance, as evidenced by antitumor activity in fluorouracil-resistant cell lines and efficacy in clinical studies in fluoropyrimidine-refractory patients.

Clinical value of trifluridine/tipiracil monotherapy

- In the pivotal Phase III RECOURSE trial in patients with chemorefractory $\mathrm{mCRC}$, trifluridine/tipiracil monotherapy prolonged overall survival by approximately 2 months versus placebo, and had acceptable tolerability, with most grade $\geq 3$ adverse events being manageable hematological events. Performance status was also maintained in these patients, as indicated by analysis of quality of life proxies.

- The efficacy profile of trifluridine/tipiracil monotherapy in third-line treatment of refractory mCRC is similar to regorafenib (the only other agent approved in the third-line setting), but it has a differing toxicity profile, with a lower frequency of hand-foot syndrome and hepatic toxicities than seen with regorafenib.

- Trifluridine/tipiracil monotherapy has a potential application in Gl cancers other than colorectal cancer, with encouraging efficacy and safety having been shown in a Phase II trial in patients with advanced gastric cancer (GC), and a Phase III trial in patients with metastatic GC is now underway.

Combinations with trifluridine/tipiracil

- Combining trifluridine/tipiracil with other anticancer agents has the potential to improve its efficacy, and clinical and preclinical studies have supported the rationale for clinical use of combinations by showing that trifluridine/tipiracil combined with other chemotherapies, VEGF and EGFR inhibitors or immune checkpoint inhibitors, enhanced antitumor activity compared with these agents alone.

- A number of clinical trials of combination regimens of trifluridine/tipiracil with irinotecan, oxaliplatin, bevacizumab, nintedanib, panitumumab, cetuximab and/or nivolumab have been completed, are underway or planned.

- Encouraging safety and preliminary efficacy data have already been obtained in Phase I/II trials of trifluridine/tipiracil with irinotecan, with oxaliplatin, with bevacizumab, with nintedanib and with panitumumab. Assessment of triple combination therapies is also underway in other clinical trials.

\section{Discussion \& future perspective}

- The clinical development plan for trifluridine/tipiracil is highly active and very promising, with positive results obtained in recent trials of the monotherapy in advanced GC and of combination therapy in earlier treatment lines for $\mathrm{mCRC}$.

- Trifluridine/tipiracil has the potential to form the chemotherapeutic backbone in the continuum of care for Gl cancers in the future.

\section{References}

Papers of special note have been highlighted as: $\bullet$ of interest; $\bullet \bullet$ of considerable interest

1. Torre LA, Bray F, Siegel RL, Ferlay J, Lortet-Tieulent J, Jemal A. Global cancer statistics, 2012. CA Cancer J. Clin. 65(2), 87-108 (2015).

2. Ferlay J, Soerjomataram I, Dikshit R et al. Cancer incidence and mortality worldwide: sources, methods and major patterns in GLOBOCAN 2012. Int. J. Cancer 136(5), e359-e386 (2015).

3. Van Cutsem E, Cervantes A, Nordlinger B, Arnold D. Metastatic colorectal cancer: ESMO clinical practice guidelines for diagnosis, treatment and follow-up. Ann. Oncol. 25(Suppl. 3), iii1-iii9 (2014).

4. Heinemann V, Von Weikersthal LF, Decker T et al. FOLFIRI plus cetuximab versus FOLFIRI plus bevacizumab as first-line treatment for patients with metastatic colorectal cancer (FIRE-3): a randomised, open-label, Phase 3 trial. Lancet Oncol. 15(10), 1065-1075 (2014).

5. Cremolini C, Loupakis F, Antoniotti C et al. FOLFOXIRI plus bevacizumab versus FOLFIRI plus bevacizumab as first-line treatment of patients with metastatic colorectal cancer: updated overall survival and molecular subgroup analyses of the open-label, Phase 3 TRIBE study. Lancet Oncol. 16(13), 1306-1315 (2015). 
6. Smyth EC, Verheij M, Allum W, Cunningham D, Cervantes A, Arnold D. Gastric cancer: ESMO clinical practice guidelines for diagnosis, treatment and follow-up. Ann. Oncol. 27(Suppl. 5), v38-v49 (2016).

7. Digklia A, Wagner AD. Advanced gastric cancer: current treatment landscape and future perspectives. World J. Gastroenterol. 22(8), 2403-2414 (2016).

8. Benson AB, 3rd, Venook AP, Cederquist L et al. Colon cancer, version 1.2017, NCCN clinical practice guidelines in oncology. J. Natl Compr. Canc. Netw. 15(3), 370-398 (2017).

9. Van Cutsem E, Cervantes A, Adam R et al. ESMO consensus guidelines for the management of patients with metastatic colorectal cancer. Ann. Oncol. 27(8), 1386-1422 (2016).

10. Yoshino T, Arnold D, Taniguchi $\mathrm{H}$ et al. Pan-Asian adapted ESMO consensus guidelines for the management of patients with metastatic colorectal cancer; a JSMO - ESMO initiative endorsed by CSCO, KACO, MOS, SSO and TOS. Ann. Oncol. 29(1), 44-70 (2017).

11. Ajani JA, D'amico TA, Almhanna K et al. Gastric cancer, version 3.2016, NCCN clinical practice guidelines in oncology. J. Natl Compr. Canc. Netw. 14(10), 1286-1312 (2016).

12. Burness CB, Duggan ST. Trifluridine/tipiracil: a review in metastatic colorectal cancer. Drugs 76(14), 1393-1402 (2016).

13. Longley DB, Harkin DP, Johnston PG. 5-Fluorouracil: mechanisms of action and clinical strategies. Nat. Rev. Cancer 3(5), 330-338 (2003).

14. Chen J, Han M, Saif MW. TAS-102: an emerging oral fluoropyrimidine. Anticancer Res. 36(1), 21-26 (2016).

15. Hirsch BR, Zafar SY. Capecitabine in the management of colorectal cancer. Cancer Manag. Res. 3, 79-89 (2011).

16. Goldberg RM, Sargent DJ, Morton RF et al. A randomized controlled trial of fluorouracil plus leucovorin, irinotecan, and oxaliplatin combinations in patients with previously untreated metastatic colorectal cancer. J. Clin. Oncol. 22(1), 23-30 (2004).

17. Yamashita F, Komoto I, Oka H et al. Exposure-dependent incorporation of trifluridine into DNA of tumors and white blood cells in tumor-bearing mouse. Cancer Chemother. Pharmacol. 76(2), 325-333 (2015).

18. Lenz HJ, Stintzing S, Loupakis F. TAS-102, a novel antitumor agent: a review of the mechanism of action. Cancer Treat. Rev. 41(9), 777-783 (2015).

-. In-depth review of the mechanism of action of trifluridine/tipiracil.

19. Sakamoto K, Yokogawa $\mathrm{T}$, Ueno $\mathrm{H}$ et al. Crucial roles of thymidine kinase 1 and deoxyUTPase in incorporating the antineoplastic nucleosides trifluridine and 2'-deoxy-5-fluorouridine into DNA. Int. J. Oncol. 46(6), 2327-2334 (2015).

20. Peters GJ. Therapeutic potential of TAS-102 in the treatment of gastrointestinal malignancies. Ther. Adv. Med. Oncol. 7(6), 340-356 (2015).

21. Zaniboni A, Bertocchi P, Barni S, Petrelli F. TAS-102 (Lonsurf $\left.{ }^{\circledR}\right)$ for the treatment of metastatic colorectal cancer. A concise review. Clin. Colorectal. Cancer 15(4), 292-297 (2016).

22. Emura T, Murakami Y, Nakagawa F, Fukushima M, Kitazato K. A novel antimetabolite, TAS-102 retains its effect on FU-related resistant cancer cells. Int. J. Mol. Med. 13(4), 545-549 (2004).

23. Mayer RJ, Van Cutsem E, Falcone A et al. Randomized trial of TAS-102 for refractory metastatic colorectal cancer. N. Engl. J. Med. 372(20), 1909-1919 (2015).

-. Pivotal Phase III trial demonstrating improved overall survival of trifluridine/tipiracil plus best supportive care versus placebo plus best supportive care in patients with refractory metastatic colorectal cancer (mCRC).

24. Yoshino T, Mizunuma N, Yamazaki K et al. TAS-102 monotherapy for pretreated metastatic colorectal cancer: a double-blind, randomised, placebo-controlled Phase 2 trial. Lancet Oncol. 13(10), 993-1001 (2012).

25. Van Cutsem E, Mayer RJ, Laurent Set al. The subgroups of the Phase III RECOURSE trial of trifluridine/tipiracil (TAS-102) versus placebo with best supportive care in patients with metastatic colorectal cancer. Eur. J. Cancer 90, 63-72 (2018).

26. Xu J, Kim TW, Shen L, et al. Results of a randomized, double-blind, placebo-controlled, Phase III trial of trifluridine/tipiracil (TAS-102) monotherapy in Asian patients with previously treated metastatic colorectal cancer: the TERRA study. J. Clin. Oncol. 36(4), 350-358 (2018).

27. Van Cutsem E, Falcone A, Garcia-Carbonero R, et al. Proxies of quality of life in metastatic colorectal cancer: analyses in the RECOURSE trial. ESMO Open 2(5), e000261 (2017).

28. Tabernero J, Van Cutsem E, Ohtsu A et al. QTWiST analysis of the RECOURSE trial of trifluridine/tipiracil in metastatic colorectal cancer. ESMO Open 2(5), e000284 (2017).

29. Masuishi $\mathrm{T}$, Taniguchi $\mathrm{H}$, Hamauchi $\mathrm{S}$ et al. Regorafenib versus trifluridine/tipiracil for refractory metastatic colorectal cancer: a retrospective comparison. Clin. Colorectal. Cancer 16(2), e15-e22 (2017).

30. Vivot A, Jacot J, Zeitoun JD, Ravaud P, Crequit P, Porcher R. Clinical benefit, price and approval characteristics of FDA-approved new drugs for treating advanced solid cancer, 2000-2015. Ann. Oncol. 28(5), 1111-1116 (2017).

31. Therasse P, Perron B, Novack SA, Abastado JP. The Servier Oncology Pipeline in 2017. Future Oncol. 13(17), 1527-1536 (2017). 
32. Bando H, Doi T, Muro K et al. A multicenter Phase II study of TAS-102 monotherapy in patients with pre-treated advanced gastric cancer (EPOC1201). Eur. J. Cancer 62, 46-53 (2016).

-. Double-blind, placebo-controlled Phase II trial reporting positive efficacy and acceptable toxicity of trifluridine/tipiracil in patients with pretreated advanced gastric cancer.

33. Ilson DH, Tabernero J, Shitara K et al. TAGS, a randomized, double-blind, Phase 3 study evaluating TAS-102 plus best supportive care vs placebo plus best supportive care in patients with metastatic gastric cancer refractory to standard treatments. J. Clin. Oncol. 34(15 Suppl.), TPS4141-TPS4141 (2016).

34. Carethers JM. Systemic treatment of advanced colorectal cancer: tailoring therapy to the tumor. Ther. Adv. Gastroenterol. 1(1), 33-42 (2008).

35. Doi T, Yoshino T, Fuse $\mathrm{N}$ et al. Phase I study of TAS-102 and irinotecan combination therapy in Japanese patients with advanced colorectal cancer. Invest New Drugs 33(5), 1068-1077 (2015).

- Phase I trial in which the recommended dose of trifluridine/tipiracil and irinotecan combination therapy was determined in Japanese patients with advanced colorectal cancer.

36. Nishina T, Kuboki Y, Shinozaki E et al. A multicentre Phase I/II study of TAS-102 with nintedanib in patients with metastatic colorectal cancer refractory to standard therapies (N-TASK FORCE: EPOC1410); Phase I results. Ann. Oncol. 27(Suppl. 6), P472-P472 (2016).

- Phase I results of a Phase I/II study on trifluridine/tipiracil in combination with nintedanib in patients with $\mathrm{mCRC}$ refractory to standard therapies.

37. Bijnsdorp IV, Kruyt FA, Gokoel S, Fukushima M, Peters GJ. Synergistic interaction between trifluorothymidine and docetaxel is sequence dependent. Cancer Sci. 99(11), 2302-2308 (2008).

38. Simkens LH, Van Tinteren H, May A et al. Maintenance treatment with capecitabine and bevacizumab in metastatic colorectal cancer (CAIRO3): a Phase 3 randomised controlled trial of the Dutch Colorectal Cancer Group. Lancet 385(9980), 1843-1852 (2015).

39. Salem ME, Wang H, Fakih M et al. An open-label, multi-center, Phase 2 study of switch maintenance with TAS-102 plus bevacizumab following oxaliplatin or irinotecan-based fluoropyrimidine-containing induction chemotherapy in patients with metastatic colorectal cancer: ALEXANDRIA study. J. Clin. Oncol. 34(15 Suppl.), TPS3624-TPS3624 (2016).

40. Baba Y, Tamura T, Satoh Y et al. Panitumumab interaction with TAS-102 leads to combinational anticancer effects via blocking of EGFR-mediated tumor response to trifluridine. Mol. Oncol. 11(8), 1065-1077 (2017).

41. Nukatsuka M, Nakagawa F, Saito H, Sakata M, Uchida J, Takechi T. Efficacy of combination chemotherapy using a novel oral chemotherapeutic agent, TAS-102, with irinotecan hydrochloride on human colorectal and gastric cancer xenografts. Anticancer Res. 35(3), 1437-1445 (2015).

42. Temmink OH, Hoebe EK, Fukushima M, Peters GJ. Irinotecan-induced cytotoxicity to colon cancer cells in vitro is stimulated by pre-incubation with trifluorothymidine. Eur. J. Cancer 43(1), 175-183 (2007).

43. Hollebecque A, Argiles G, Andre T et al. A Phase I dose-escalation of trifluridine/tipiracil in combination with oxaliplatin in metastatic colorectal cancer. J. Clin. Oncol. 35(15 Suppl.), TPS3626-TPS3626 (2017).

44. Kuboki Y, Nishina T, Shinozaki E et al. TAS-102 plus bevacizumab for patients with metastatic colorectal cancer refractory to standard therapies (C-TASK FORCE): an investigator-initiated, open-label, single-arm, multicentre, Phase 1/2 study. Lancet Oncol. 18(9), 1172-1181 (2017).

-• Phase I/II trial demonstrating positive activity and manageable safety of trifluridine/tipiracil in combination with bevacizumab in patients with $\mathrm{mCRC}$ refractory to standard therapies.

45. Moiseenko V, Lesniewski-Kmak K, Saunders MP et al. Design of a Phase II study evaluating trifluridine/tipiracil+bevacizumab and capecitabine+bevacizumab for first-line treatment of patients with unresectable metastatic colorectal cancer (mCRC) who are non-eligible for intensive therapy (TASCO1). Presented at: ESMO 19th World Congress on Gastrointestinal Cancer. Barcelona, Spain, 28 June-1 July 2017.

46. Kato T, Kagawa Y, Komatsu Y et al. A Phase I/II study for panitumumab combined with TAS-102 in patients with RAS wild-type metastatic colorectal cancer (APOLLON study): Phase I results. J. Clin. Oncol. 35(4 Suppl.), 770-770 (2017).

- Phase I results of a Phase I/II trial on trifluridine/tipiracil in combination with panitumumab in patients with RAS wild-type mCRC.

47. Alcindor T, Beauger N. Oxaliplatin: a review in the era of molecularly targeted therapy. Curr. Oncol. 18(1), 18-25 (2011).

48. Temmink OH, Hoebe EK, Van Der Born K, Ackland SP, Fukushima M, Peters GJ. Mechanism of trifluorothymidine potentiation of oxaliplatin-induced cytotoxicity to colorectal cancer cells. Br. J. Cancer 96(2), 231-240 (2007).

49. Nukatsuka M, Nakagawa F, Takechi T. Efficacy of combination chemotherapy using a novel oral chemotherapeutic agent, TAS-102, with oxaliplatin on human colorectal and gastric cancer xenografts. Anticancer Res. 35(9), 4605-4615 (2015).

50. Rosen LS, Jacobs IA, Burkes RL. Bevacizumab in colorectal cancer: current role in treatment and the potential of biosimilars. Target Oncol. 12(5), 599-561 (2017). 
51. Tsukihara H, Nakagawa F, Sakamoto K et al. Efficacy of combination chemotherapy using a novel oral chemotherapeutic agent, TAS-102, together with bevacizumab, cetuximab, or panitumumab on human colorectal cancer xenografts. Oncol. Rep. 33(5), 2135-2142 (2015).

- Preclinical investigations demonstrating enhanced antitumor effects of trifluridine/tipiracil when combined with bevacizumab, cetuximab or panitumumab in colorectal cancer xenografts.

52. Suzuki N, Nakagawa F, Matsuoka K, Takechi T. Effect of a novel oral chemotherapeutic agent containing a combination of trifluridine, tipiracil and the novel triple angiokinase inhibitor nintedanib, on human colorectal cancer xenografts. Oncol. Rep. 36(6), 3123-3130 (2016).

53. Kanwar SS, Nautiyal J, Majumdar AP. EGFR(S) inhibitors in the treatment of gastro-intestinal cancers: what's new? Curr. Drug Targets 11(6), 682-698 (2010).

54. Townsley CA, Major P, Siu LL et al. Phase II study of erlotinib (OSI-774) in patients with metastatic colorectal cancer. Br. J. Cancer 94(8), 1136-1143 (2006).

55. Bijnsdorp IV, Kruyt FA, Fukushima M, Smid K, Gokoel S, Peters GJ. Molecular mechanism underlying the synergistic interaction between trifluorothymidine and the epidermal growth factor receptor inhibitor erlotinib in human colorectal cancer cell lines. Cancer Sci. 101(2), 440-447 (2010).

56. Paul B, O’Neil BH, McRee AJ. Checkpoint inhibition for colorectal cancer: progress and possibilities. Immunotherapy 8(6), 693-704 (2016).

57. Zhang CM, Lv JF, Gong L et al. Role of deficient mismatch repair in the personalized management of colorectal cancer. Int. J. Environ. Res. Public Health 13(9), 892 (2016).

58. Limagne E, Nuttin L, Spill A et al. P-256trifluridine/tipiracil combined to oxaliplatin sensitizes microsatellite stable colorectal cancer to anti-PD-1 blockade. Ann. Oncol. 28(Suppl. 3), mdx261.254-mdx261.254 (2017).

59. Suzuki N, Tsukihara H, Nakagawa F, Kobunai T, Takechi T. Synergistic anticancer activity of a novel oral chemotherapeutic agent containing trifluridine and tipiracil in combination with anti-PD-1 blockade in microsatellite stable-type murine colorectal cancer cells. Am. J. Cancer Res. 7(10), 2032-2040 (2017).

60. Lyseng-Williamson KA, Burness CB, Duggan ST. Trifluridine/tipiracil in metastatic colorectal cancer: a guide to its use. Drugs Ther. Perspect. 33(3), 110-118 (2017).

61. Borelli B, Zucchelli G, Rossini D et al. A retrospective study of trifluridine/tipiracil in pretreated metastatic colorectal cancer patients in clinical practice. Colorectal Cancer doi:10.2217/crc-2017-0023 (2018) (Epub ahead of print).

62. Loupakis F, Lonardi S. New perspectives for TAS-102: TASK successful? Lancet Oncol. 18(9), 1139-1141 (2017). 
